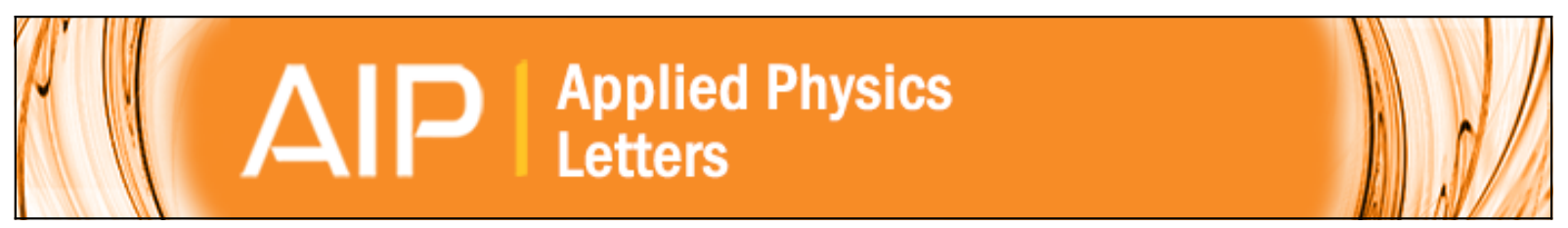

\title{
Nature of acoustic nonlinear radiation stress
}

Guillermo Rus

Citation: Applied Physics Letters 105, 121904 (2014); doi: 10.1063/1.4894827

View online: http://dx.doi.org/10.1063/1.4894827

View Table of Contents: http://scitation.aip.org/content/aip/journal/apl/105/12?ver=pdfcov

Published by the AIP Publishing

\section{Articles you may be interested in}

Simulation of nonlinear Westervelt equation for the investigation of acoustic streaming and nonlinear propagation effects

J. Acoust. Soc. Am. 134, 3931 (2013); 10.1121/1.4821201

Fast acoustic streaming in standing waves: Generation of an additional outer streaming cell

J. Acoust. Soc. Am. 134, 1791 (2013); 10.1121/1.4817888

Numerical study of nonlinear streaming inside a standing wave resonator

AIP Conf. Proc. 1474, 87 (2012); 10.1063/1.4749305

Viscous effects on the interaction force between two small gas bubbles in a weak acoustic field

J. Acoust. Soc. Am. 111, 1602 (2002); 10.1121/1.1459466

Focusing of nonlinear waves emitted from a source of large aperture angle

AIP Conf. Proc. 524, 221 (2000); 10.1063/1.1309209

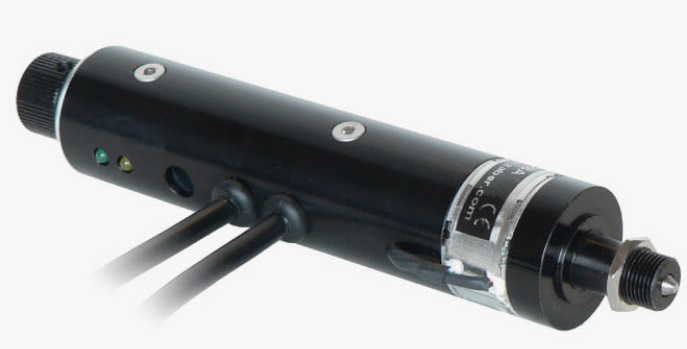

Automate your set-up with 


\title{
Nature of acoustic nonlinear radiation stress
}

\author{
Guillermo Rus ${ }^{\text {a) }}$ \\ Nondestructive Evaluation Laboratory, Department of Structural Mechanics, University of Granada, \\ Politecnico de Fuentenueva, Granada 18071, Spain
}

(Received 8 May 2014; accepted 22 August 2014; published online 23 September 2014)

\begin{abstract}
When a fluid is insonified with ultrasound, a flow consequence of a net stress becomes observable, which has been described as acoustic streaming, quartz wind, acoustic radiation force, or acoustic fountain. Following Sir James Lighthill's formulation of the Reynold's streaming, these phenomena have been attributed to a cumulative viscous effect. Instead, a multiscale effect, whereby the constitutive elastic nonlinearity scales from the ultrasonic to the macroscopic time, is here proposed and formulated to explain its origin. This raises an additional term in the NavierStokes equation, which ultimately stems from the anharmonicity of the atomic potential. In our experimental validation, this theory is consistent in water and for a range of ultrasonic configurations, whereas the formerly established viscous theory fails by an order of magnitude. This ultrasonicfluid interaction, called nonlinear mechanical radiation since it is able to remotely exert a stress field, correctly explains a wide range of industrial and biomedical active ultrasonic uses including jet engines, acoustic tweezers, cyanobacteria propulsion mechanisms, nanofluidics, or acoustic radiation force elastography. (C) 2014 AIP Publishing LLC. [http://dx.doi.org/10.1063/1.4894827]
\end{abstract}

The first experimental observations of fluid flow caused by a fast oscillatory movement were the air currents observed by Rayleigh ${ }^{1}$ in a Kundt's tube-a transparent pipe where fine powder sedimentation demonstrates the standing waves-, and the discovery by Savart of the movement of powder across the vibration nodes on Chladni's vibrating plate experiment. The advent of quartz piezoelectric sound generators in air caused a rediscovery of the phenomenon, which was then labelled quartz wind. Lord Rayleigh was possibly first to describe the experimental observation for air in $1883:^{1}$ "when the corresponding fork, strongly excited, was held to the mouth [of the Helmholtz resonator] a wind of considerable force issued from the nipple at the opposite side. This effect may rise to such intensity as to blow out a candle upon whose wick the stream is directed." These observations triggered research to try to explain them. Lord Rayleigh laid the first theoretical foundations stating that these currents could not arise in the absence of friction. However, his attempts to quantitatively explain the phenomenon failed.

In 1948, Eckart ${ }^{2}$ derived the net radiation pressure and ensuing fluid acceleration caused solely by the viscous energy deposition during sound wave propagation. The following year, his colleague Liebermann ${ }^{3}$ documented the first quantitative experimental measurements of the so-called Reynolds streaming, with the intention of using Eckart's model to measure the shear and volumetric viscosity of liquids, whose results were soon questioned. ${ }^{4}$ Not much later, Nyborg ${ }^{5}$ concluded in his review that streaming measurements cannot be used to distinguish between absorption mechanisms.

Fifteen days later, Westervelt ${ }^{6}$ submitted from the same department and to the same journal a formulation of the phenomenon including some corrections to Eckart's original formulation. Lighthill ${ }^{7}$ generalized the formulation $25 \mathrm{yr}$ later

a) grus@ugr.es; http://www.ugr.es/ grus including energy streams, where the inertia is relevant and unifying the reverse effect of sound generation aerodynamically by flows interacting with boundaries. This formulation was later revisited by Riley ${ }^{8,9}$ and Tjøtta, ${ }^{10,11}$ formally adopting the formulation of Reynolds streaming, defined as the mean value of the acoustic momentum flux. Langevin's radiation theory differs from Rayleigh's on the experimental setup, as clarified by Hasegawa: ${ }^{12}$ radiation pressure is defined as the time-averaged stress measured either on the insonicated wall of a closed vessel according to Rayleigh or on an obstacle in the path of the acoustic beam, according to Langevin.

In essence, the mechanism described by the above formulation is the following. Given a harmonic oscillation with zero mean, the viscous energy deposition is the sole source of acoustic energy flux that permits gradients in momentum flux that contributes to the Reynolds streaming. In other words, a nonzero time averaged Reynolds stress is built up to balance the average of the viscous attenuation of the propagating wave, and this stress pushes the fluid in the direction of the wave propagation. The attribution of viscous Reynolds streaming to the observed acoustic fountain and quartz wind effects seems to have been adopted gradually based on the mentioned literature.

A variety of experimental measurement techniques have recently been applied to measure streaming in viscous fluids. Starritt and Humphrey ${ }^{13}$ applied anemometry and ultrasonic doppler to measure the streaming produced by an immersed medical probe. Nowicki et al. ${ }^{14}$ performed ultrasonic Doppler velocimetry to measure the acoustic streaming velocity in water with corn starch, in order to compare it to the theory by Starritt. Particle image velocimetry has also been used by Cosgrove et al. ${ }^{15}$ to measure acoustic streaming velocity of commercial echographic transducers. ${ }^{16}$

The scarcity of conclusive matching between experiments and theory motivated the measurements presented here. Unexpectedly, parameters describing the acoustic 


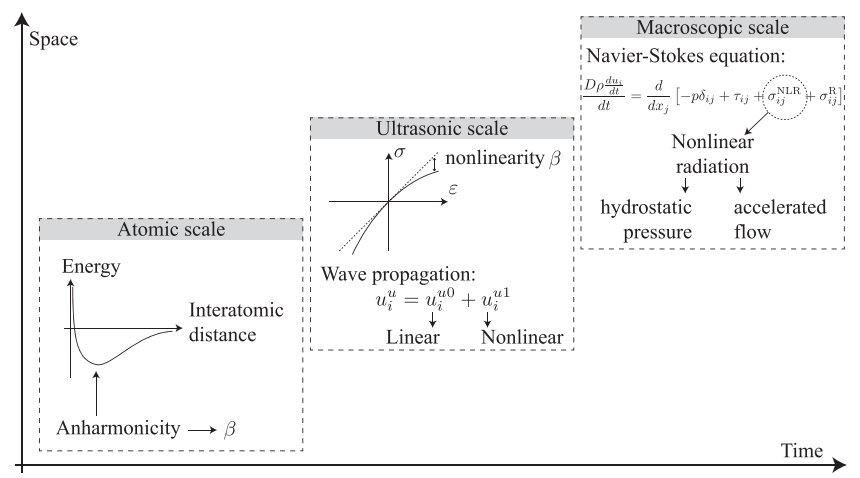

FIG. 1. Cascade of cause-effect mechanisms, from the atomic to the macroscopic scale.

fountain of orders of magnitude stronger than predicted by the theory above were measured. Here, we propose a multiscale theory that stems from the constitutive nonlinearity, in the sense of deviation from Hooke's law, rooted in the anharmonicity of the atomic potential. The theory formulates an additional macroscopic stress term in the Navier-Stokes equations that arises from scaling up the constitutive nonlinearity at the ultrasonic time scale. The theory is formulated in the following paragraphs based on the concepts of constitutive nonlinearity, viscosity, and multiscale mechanics, while the experimental validation is presented later.

A full understanding and modeling of acoustic streaming is useful for a wide range of industrial and medical existing and potential applications. Aeroacoustics of jet engines, acoustic tweezers, and uses of ultrasonics as active elements are just a few applications where nonlinear radiation (NLR) is a core mechanism. As Nyborg ${ }^{5}$ already pointed out, a significant number of biological processes are being explained by acoustic streaming, ${ }^{17}$ including cyanobacteria propulsion mechanisms, yeast mechanisms, or hearing mechanisms by the cochlea. ${ }^{18}$ Applications to medical engineering have also been envisaged recently, for instance, for nanofluidics, ${ }^{19-22}$ acoustic tweezers, or acoustic radiation force elastography, ${ }^{23}$ where efforts are currently required in accurately simulating the exerted radiation force responsible for generating the shear waves that propagate through tissue and correctly quantify its shear modulus.

Before deriving the equations, a rough picture of the origin, in terms of the cascade of cause-effect mechanisms at different scales, is depicted in Fig. 1. The symbols will be properly defined below.

The origin of the mechanism stems from the constitutive nonlinearity of the fluid, characterized by $\beta$, which is a consequence of the atomic potential anharmonicity.

This atomic property scales up to the ultrasonic scale, of the order of the micrometer and microsecond, where the propagation $u_{i}^{u}$ of the ultrasonic waves in presence of constitutive nonlinearity generates additional motion modes at higher frequencies $u_{i}^{u 1}$.

Finally, this type of propagation scales to the macroscopic scale by building up an additional stress term $\sigma_{i j}^{\mathrm{NLR}}$ to the Navier-Stokes equation that manifests at time and space scales above the ultrasonic period and the wavelength. This radiation stress creates two observable effects: (i) it is a source of hydrostatic pressure that increases the piezometric level, which is particularly observable at the fluid free surface in the form of a protuberance and (ii) independently, in case the stress field has a gradient, it builds up a flow acceleration observable as a streaming.

Matter is composed of atoms or molecules that maintain a volume due to the balance between attractive and repulsive forces that the particles exert into each other. These interatomic and intermolecular forces are represented by the potential energy, which is the sum of repulsive and attractive contributions that depend on the type of bond. A typical form $^{24}$ is approximated by the repulsive and attractive contributions $U=\frac{A}{r^{12}}-\frac{B}{r^{6}}$ that depend on the internuclear distance $r$.

The spatial structure at rest is defined by the internuclear distance $r^{0}$ that minimizes the potential energy $U$. If a pressure $p$ is applied, its resultant force $f$ over each bond is equilibrated by an energy potential gradient $f=\frac{d U}{d r}$ induced by a change $\Delta r=r-r^{0}$ in the separation $r$. The dependency can be evidenced by expanding $U(r)$ around the equilibrium distance $r^{0}$

$$
\begin{aligned}
U\left(r_{0}+\Delta r\right)= & U\left(r_{0}\right)+\underbrace{\left.\frac{d U}{d r}\right|_{r=r_{0}} \Delta r}_{\text {zero at equilibrium } f\left(r^{0}\right)=0} \\
& +\underbrace{\left.\frac{1}{2 !} \frac{d^{2} U}{d r^{2}}\right|_{r=r_{0}} \Delta r^{2}}_{\text {harmonic potential }}+\left.\frac{1}{3 !} \frac{d^{3} U}{d r^{3}}\right|_{r=r_{0}} \Delta r^{3}+\text { h.o.t. }
\end{aligned}
$$

Given that the change of volume due to a hydrostatic pressure $p$ depends on the internuclear distance $\frac{\Delta V}{V}=\varepsilon_{k k}=\frac{3 \Delta r}{r}$, pressure can be expressed in terms of the potential energy

$$
\begin{aligned}
-p= & \frac{f}{\text { area }} \sim \frac{f}{r^{2}}=\left.\frac{1}{3 r} \frac{d^{2} U}{d r^{2}}\right|_{r=r_{0}} \varepsilon_{k k}+\left.\frac{1}{18} \frac{d^{3} U}{d r^{3}}\right|_{r=r_{0}} \varepsilon_{k k}^{2} \\
& +\left.\frac{r}{162} \frac{d^{4} U}{d r^{4}}\right|_{r=r_{0}} \varepsilon_{k k}^{3}+\text { h.o.t. }
\end{aligned}
$$

The first term of this expansion matches the definition of compressibility, which is linked to the harmonic part of the potential, but also explains the nonlinear behavior of the compressibility by

$$
\begin{aligned}
& -p=K \varepsilon_{k k}+\beta K \varepsilon_{k k}^{2}+\gamma K \varepsilon_{k k}^{3}+\text { h.o.t. } \Longleftrightarrow \\
& K=\left.\frac{1}{3 r} \frac{d^{2} U}{d r^{2}}\right|_{r=r_{0}}, \quad \beta=\left.\frac{r}{6} \frac{\frac{d^{3} U}{d r^{3}}}{\frac{d^{2} U}{d r^{2}}}\right|_{r=r_{0}}, \quad \gamma=\left.\frac{r^{2}}{54} \frac{\frac{d^{4} U}{d r^{4}}}{\frac{d^{2} U}{d r^{2}}}\right|_{r=r_{0}}
\end{aligned}
$$

If the typical potential energy form $U=\frac{A}{r^{12}}-\frac{B}{r^{6}}$ is adopted, the equilibrium distance is $r^{0}=2^{\frac{1}{6}} A^{\frac{1}{6}} B^{\frac{-1}{6}}$ and the nonlinear compressibility constitutive law is linked to the interatomic forces by

$$
K=3 \sqrt{2} A^{\frac{-3}{2}} B^{\frac{5}{2}}, \quad \beta=-7 / 2=-3.5, \quad \gamma=371 / 54 \approx 6.87 .
$$

The latter value of $\beta$ is compatible with recent ultrasonicsbased measurements in water. ${ }^{25,26}$

If small deformations are assumed and plastic flow neglected, the following elasticity governing equations that 
TABLE I. Continuum mechanics variables.

\begin{tabular}{lcc}
\hline \hline Quantity & Symbol & Units \\
\hline Space (cartesian) & $x_{i}$ & $\mathrm{~m}$ \\
Time & $t$ & $\mathrm{~s}$ \\
Displacement & $u_{i}$ & $\mathrm{~m}$ \\
Velocity & $\dot{u}_{i}=\frac{d u_{i}}{d t}$ & $\mathrm{~m} / \mathrm{s}$ \\
Velocity rate & $\frac{D \dot{u}_{i}}{D t}=\frac{\partial \dot{u}_{i}}{\partial t}+\dot{u}_{j} \dot{u}_{i, j}$ & $\mathrm{~m} / \mathrm{s}^{2}$ \\
Strain & $\varepsilon_{i j}$ & - \\
Stress & $\sigma_{i j}$ & $\mathrm{~Pa}$ \\
Pressure & $p$ & $\mathrm{~Pa}$ \\
Body force & $b_{i}^{\mathrm{m}}$ & $\mathrm{N} / \mathrm{kg}$ \\
Lamé constants & $\lambda, \mu$ & $\mathrm{Pa}$ \\
Density & $\rho$ & $\mathrm{Kg} / \mathrm{m}^{3}$ \\
Shear, volumetric viscosity & $\eta, \eta^{v}$ & $\mathrm{~Pa} \mathrm{~s}=\mathrm{kg} / \mathrm{ms}$ \\
\hline \hline
\end{tabular}

relate the quantities in Table I are the starting point of the detailed derivation provided in the supplementary material ${ }^{27}$ and summarized here: the equilibrium, compatibility and constitutive equations, respectively,

$$
\frac{D\left(\rho \dot{u}_{i}\right)}{D t}=\sigma_{i j, j}+\rho b_{i}^{\mathrm{m}} \quad \varepsilon_{i j}=\frac{1}{2}\left(u_{i, j}+u_{j, i}\right) \quad \sigma_{i j}=\sigma_{i j}\left(\varepsilon_{k l}\right) .
$$

The linear elastic dependency is enriched with quadratic terms, following the series expansion concept put forth by Landau. ${ }^{28}$ Only the volumetric part is detailed in terms of the nonlinearity parameter $\beta$ due to the simplicity of the volumetric strain $\operatorname{vol}\left(\varepsilon_{i j}\right)$ being scalar,

$$
\begin{aligned}
& -p=-3 K \operatorname{vol}\left(\varepsilon_{i j}\right)+9 \beta K \operatorname{vol}\left(\varepsilon_{i j}\right)^{2}-3 \eta^{v} \operatorname{vol}\left(\dot{\varepsilon}_{i j}\right) \\
& \tau_{i j}=\underbrace{2 \mu \operatorname{dev}\left(\varepsilon_{i j}\right)}_{\text {Linear elastic }} \underbrace{2 \eta \operatorname{dev}\left(\dot{\varepsilon}_{i j}\right)}_{\text {Nonlinear elastic }} \text {, }
\end{aligned}
$$

where stress and strain tensors are split into volumetric (scalar) and deviatoric (tensor) parts by,

$$
\begin{aligned}
\sigma_{i j} & =\underbrace{-p \delta_{i j}}_{\text {volumetric }}+\underbrace{\tau_{i j}}_{\text {deviatoric }}, \quad p=-\frac{1}{3} \sigma_{k k}, \\
\varepsilon_{i j} & =-\operatorname{vol}\left(\varepsilon_{i j}\right) \delta_{i j}+\operatorname{dev}\left(\varepsilon_{i j}\right), \quad \operatorname{vol}\left(\varepsilon_{i j}\right)=-\frac{1}{3} \varepsilon_{k k} .
\end{aligned}
$$

A nondimensionalization ${ }^{29}$ of the magnitudes with respect to the ultrasonic scale will make second order terms become evident and allow to split the ultrasonic from the macroscopic scales,

$$
u_{i}^{*}\left(x_{i}, t\right)=u_{i}^{s}\left(x_{i}^{s}, t^{s}\right)+u_{i}^{u}\left(x_{i}^{u}, t^{u}\right)
$$

where the slow scale solution $u^{s}$ is specified as independent of the ultrasonic scale $u^{u}$ by defining the former as the average of $u$ over an ultrasonic spatial and temporal cycle (i.e. reference ultrasonic wavelength and period). The multiscale method $^{30}$ is a strategy for capturing the long-term effects that are not visible in the small scale but become significant at a larger and slower scale by adding a so-called secular term to the differential equation. In this case, the secular terms become the Reynolds stress and the nonlinear radiation.
The ensuing nondimensional motion equations can be split by the multiscale technique into two coupled problems at ultrasonic and slow scales respectively. The general form of Navier-Stokes equation is recovered from the latter, where two additional source terms arise on the slow-scale NavierStokes equation from the multiscale secular terms: the general form of the well-known Reynolds stress $\sigma_{i j}^{\mathrm{R}}$ and the nonlinear radiation stress $\sigma_{i j}^{\mathrm{NLR}}$,

$$
\begin{aligned}
\frac{D \rho \frac{d u_{i}}{d t}}{d t} & =\frac{d}{d x_{j}}\left[-p \delta_{i j}+\tau_{i j}+\sigma_{i j}^{\mathrm{NLR}}+\sigma_{i j}^{\mathrm{R}}\right] \\
& \approx-\frac{d p}{d x_{i}}+\frac{d \tau_{i j}}{d x_{j}}+(-\beta+1) K \alpha\left|\varepsilon^{u}\right|^{2} \xi_{i},
\end{aligned}
$$

where the nonlinear radiation stress generalizes in the form,

$$
\sigma_{i j}^{\mathrm{NLR}}=2 K \delta_{i j} \sum_{n=1}^{\infty} \beta_{2 n}(-1)^{n}\left|\frac{\varepsilon^{u}}{2}\right|^{2 n} e^{-2 n \alpha \xi_{k} x_{k}}
$$

A particularly visible manifestation of the NLR theory is predicted when excited opposite to gravity, i.e., upwards, and close to the fluid surface. Two phenomena formulated below are combined to generate what is usually called acoustic fountain. First, the nonlinear radiation pressure inside the beamshaped ultrasonicated volume raises its piezometric level generating a visible column of fluid emanating from its free surface. The piezometric level $h=\frac{p}{\rho g}$ is due to the hydrostatic pressure $p=-\frac{1}{3} \sigma_{k k}$. Second, the pressure gradient accelerates the volume against the gravity moving the fluid column in a manner that recalls a fountain. The net acceleration comes from the gradient, $a_{i}=\frac{1}{\rho} \frac{d \sigma_{i j}^{\mathrm{NLR}+\mathrm{R}}}{d x_{j}}$. Note that the column consequence of the piezometric level exists without the need for any flow, and therefore independently of the second phenomenon of pressure gradient acceleration. What can be measured using this setup is (1) the piezometric height $h$ of the fluid column, (2) the flow velocity $v$, and (3) the ultrasonic pressure $p^{u}$ inside it.

The model is tested against observations by comparing its measurable values and their dependencies when changing the following parameters, in order to validate the model over a range of configurations and simultaneously discard any dependency on the experimental design: (1) ultrasonic power $\varepsilon^{u}, p^{u}=\rho c^{2} \varepsilon^{u}$, (2) ultrasonic frequency $\omega=2 \pi f$, (3) time modulation $\varepsilon^{u}(x, t)=\varepsilon^{u} f(x) m(t)$ by pulse width modulation defined by a duty cycle $d$.

A PZT-5 piezoelectric ceramic $0.5 \mathrm{~mm}$ thick, of area $50 \mathrm{~mm}^{2}$ and a resonant frequency of $4.15 \mathrm{MHz}$ is used to generate a vertical ultrasonic beam from $40 \mathrm{~mm}$ beneath the degassed water surface (to avoid the near field) in an immersion tank, following the scheme in Fig. 2. The transducer is excited with a monochromatic sine signal of variable frequency $\omega=2 \pi f$ modulated by a duty cycle $d$ and a repetition rate of $1 \mathrm{~ms}$ generated with an Agilent 33250 generator and amplified with an Amplifier Research 150A100B (150 W, $10 \mathrm{kHz}-100 \mathrm{MHz})$ amplifier at $46 \mathrm{~dB}(200 \times)$ gain programmed to generate a variable amplitude of $20-400 \mathrm{~V}$, so that the peak ultrasonic pressure ranges up to $p^{u}=600 \mathrm{kPa}$. The ultrasonic pressure $p^{u}$, frequency $f$, and duty cycle $d$ are varied to discard any dependency on the hardware and experimental design within the range of operation. 


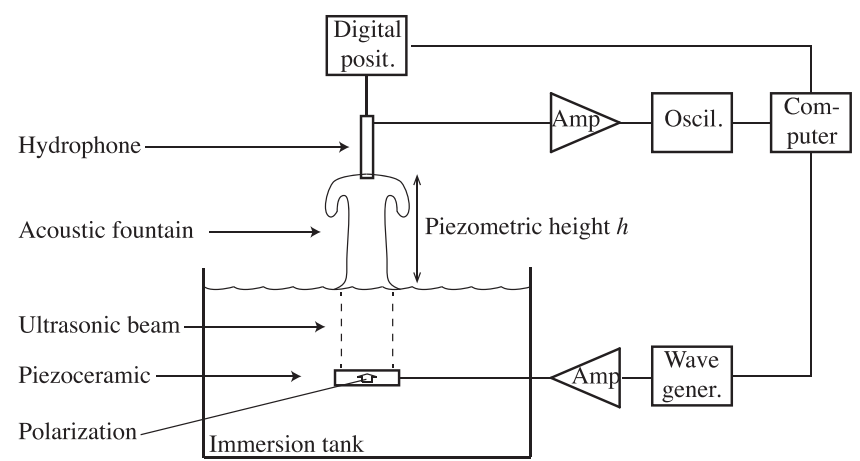

FIG. 2. Scheme of the experimental setup.

The height $h$ of the generated acoustic fountain (see Fig. 3) and the ultrasonic pressure $p^{u}$ inside its top are probed by a hydrophone HGL (Onda Corp.) conditioned with a Panametrics preamplifier at $37.5 \mathrm{~dB}(75 \times)$ gain moving along the fountain axis by a digital controlled positioning system (NDT automation). The signal is then digitized with a 12 bits $320 \mathrm{MHz}$ Acquiris digitizer using 500× averaging. The digitized signal is gated to capture exactly 40 stable cycles to allow a numerically error-free FFT.

The first approximation of the solution of the NS equations on this beam becomes straightforward by assimilating the beam to a variable-section pipe where the flow is derived by imposing the NLR acceleration.

An ultrasonic piezoelectric generator is immersed in fluid and excited to propagate towards axis $a, \xi_{i}=\mathbf{e}_{a}$ (e.g., $\mathbf{e}_{3}=(0$, $0,1)$ for the vertical case) a continuous beam-shaped field, as a first approximation, whose amplitude $\varepsilon^{u}\left(x_{a}\right)=\varepsilon^{u} f\left(x_{a}\right)$ decreases monotonically with distance beyond the so-called ultrasonic near field. This decrease is due to viscous attenuation combined with geometric dispersion and other phenomena, and may be approximated by $f\left(x_{a}\right) \approx e^{-\alpha^{\prime} x_{a}}, \alpha^{\prime}>\alpha$. Such a propagation can be formulated as

$$
u_{i}^{0}(x, t)=\frac{k \varepsilon^{u} f\left(x_{a}\right)}{2} \mathbf{e}_{a}\left(e^{i\left(\omega x_{a} / c-\omega t\right)}+e^{-i\left(\omega x_{a} / c-\omega t\right)}\right)
$$

which yields the following nonlinear radiation and Reynolds stresses, hydrostatic pressures-which are responsible for the piezometric height $h$ observed in the experiment by $p=\rho g h$-and the body forces-responsible for exerting an acceleration to the flow

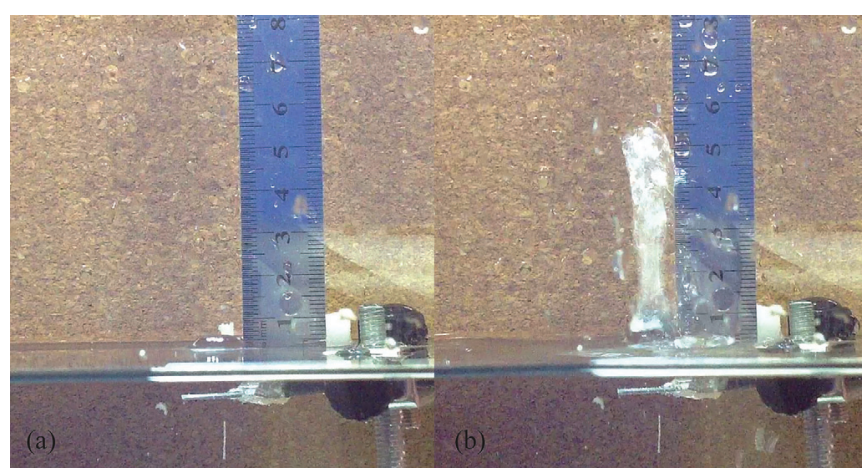

FIG. 3. Images of the generated acoustic fountains at low energy (a) and high energy (b).

$$
\begin{gathered}
\sigma_{i j}^{\mathrm{NLR}} \approx \frac{\beta K}{2} \varepsilon^{u}\left(x_{a}\right)^{2} \delta_{i j}, \quad p^{\mathrm{NLR}} \approx \frac{\beta K}{2} \varepsilon^{u}\left(x_{a}\right)^{2}, \\
\frac{d \sigma_{i j}^{\mathrm{NLR}}}{d x_{j}} \approx \beta K \varepsilon^{u 2} f\left(x_{a}\right) \frac{d f\left(x_{a}\right)}{d x_{a}} \mathbf{e}_{a}, \\
\sigma_{i j}^{\mathrm{R}}=-\frac{\rho c^{2}}{2} \varepsilon^{u}\left(x_{a}\right)^{2} \mathbf{e}_{a} \otimes \mathbf{e}_{a}, \quad p^{\mathrm{R}}=-\frac{K}{6} \varepsilon^{u}\left(x_{a}\right)^{2}, \\
\frac{d \sigma_{i j}^{\mathrm{R}}}{d x_{j}} \approx K \alpha^{\prime} \varepsilon^{u 2} e^{-2 \alpha^{\prime} x_{a}} \mathbf{e}_{a} .
\end{gathered}
$$

The ensuing fluid velocity accumulated over a distance $d$ by the former acceleration inside the ultrasonic beam, neglecting viscosity and $3 \mathrm{D}$ effects, becomes

$$
\begin{aligned}
v^{\mathrm{NLR}+\mathrm{R}}(d) & =\sqrt{2 d a}=\sqrt{2 d \frac{1}{\rho} \frac{d \sigma_{i j}^{\mathrm{NLR}+\mathrm{R}}}{d x_{j}}} \\
& \approx \sqrt{2 d(1-\beta) \alpha^{\prime} c^{2}{\overline{\varepsilon^{u}}}^{2}} .
\end{aligned}
$$

As an illustrative example of the prediction of the hydrostatic pressure phenomenon, the ultrasonic pressure that generates a $10 \mathrm{~mm}$ piezometric height using a $95 \%$ duty cycle is experimentally estimated from Fig. 4 as $350 \pm 70 \mathrm{kPa}$. In comparison, the Nonlinear radiation model (NLR, Eq. (8) (The value of $\beta=-3.5 \pm 20 \%$ is taken from the literature. $^{25,26}$ ) predicts $348 \mathrm{kPa}$, whereas the formerly believed viscosity-based Reynolds streaming (R, Eq. (9)) predicts $1181 \mathrm{kPa}$. However, the following paragraph infers the plausibility of each theory according to the complete experimental data using a formal Bayesian probability logic.

The prediction of the second effect of the fluid flow has a limited validity due to the high uncertainty in the attenuation $\alpha^{\prime}$ ( $\alpha^{\prime}$ is estimated on average as $2[\mathrm{np} / \mathrm{m}]$ from our measurements of the evolution of the ultrasonic pressure along the ultrasonic beam up to $80[\mathrm{~mm}]$ with the same equipment.) and to the variability of the particle image velocimetry measurements due to the effects of the surrounding water around the ultrasonic beam. However, some illustrative figures are provided in Table II, where particle image velocimetry observations are compared to the predictions of the Nonlinear radiation model (Eq. (10)) and the Reynolds stress model (letting $\beta=0$ ). The ultrasonic beam was generated at 4.15 MHz with $100 \%$ duty cycle for three different ultrasonic pressures, and the velocity was measured at a distance $d=20 \mathrm{~mm}$ from the transducer, before friction prevails.

Bayes' theorem allows to rank the plausibility of the hypothesized models $H_{j}\left(y=g_{j}(p) ; j=1\right.$ nonlinear radiation, $j=2$ Reynolds stress) updated by experimental observations of acoustic fountain heights $Y=y_{i}$ from independent configurations $P=p_{i}=\{f, p, d\}_{i} \in \mathcal{P}$

$$
p\left(H_{j} \mid Y\right)=\frac{p\left(Y \mid H_{j}\right) p\left(H_{j}\right)}{p(Y)}=\frac{p\left(Y \mid H_{j}\right) p\left(H_{j}\right)}{\sum_{j} p\left(Y \mid H_{j}\right) p\left(H_{j}\right)},
$$

where $p\left(Y \mid H_{j}\right)$, usually called evidence, is the marginal joint likelihood

$$
p\left(Y \mid H_{j}\right)=\int_{\mathcal{P}} p\left(Y \mid P, H_{j}\right) p\left(P \mid H_{j}\right) d P=\Pi_{i} p\left(y_{i} \mid p_{i}, H_{j}\right) p\left(p_{i} \mid H_{j}\right),
$$




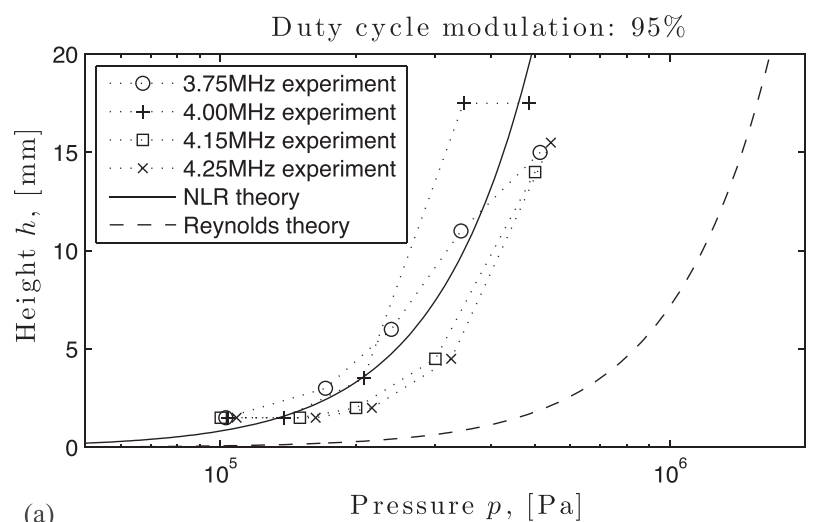

(a)

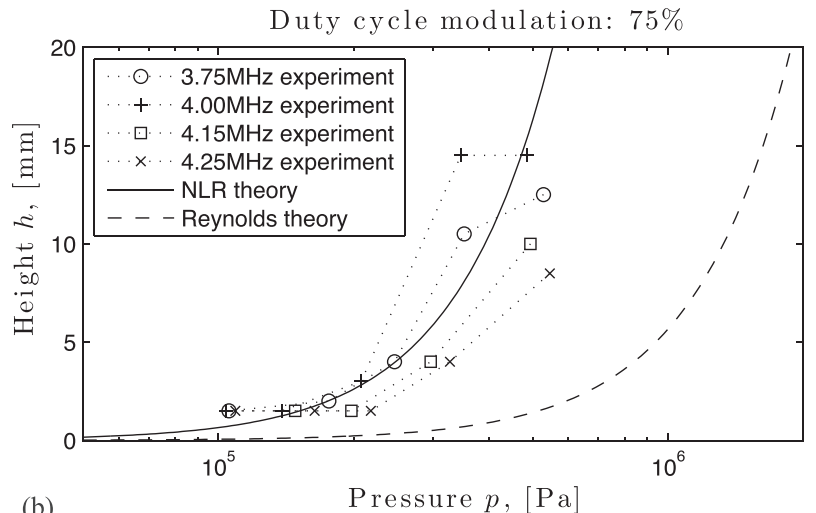

(b)

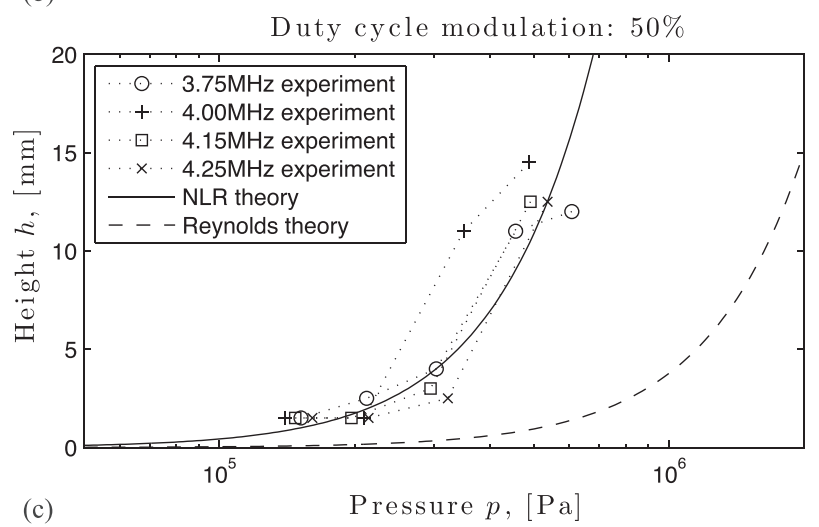

FIG. 4. Acoustic fountain height generated experimentally by an increasing ultrasonic pressure, for various frequencies and a duty cycle of $95 \%$ (a), $75 \%$ (b), and $50 \%$ (c). Superposed predictions by the NLR and Reynolds stress (R) model.

where $p\left(H_{j}\right)$ and $p\left(P \mid H_{j}\right)$ are the prior information on hypothesis and configurations, assumed equiprobable and therefore canceling constants, and the likelihood is $p\left(y_{i} \mid p_{i}, H_{j}\right)=c e^{-\frac{1}{2} \sum_{i k}\left(y_{i}-g_{j}\left(p_{i}\right)\right) \sigma^{-1}\left(y_{k}-g_{j}\left(p_{k}\right)\right)}$ assuming Gaussian experimental errors $\sigma$.

Evaluating Eq. (11) for the observations $y_{i}$ in Fig. 4 corresponding to 56 configurations $p_{i}=\{f, p, d\}_{i}$ (4 frequencies $f \times 4$ or 5 pressures $p \times 3$ duty cycles $d$ ), estimating the error

TABLE II. Particle image velocimetry measurement against predictions of the nonlinear radiation and Reynolds stress models at increasing ultrasonic pressures, at $d=20 \mathrm{~mm}$.

\begin{tabular}{lcccc}
\hline \hline$p^{u}(\mathrm{kPa})$ & $\varepsilon^{u}\left(\times 10^{-6}\right)$ & $v^{\mathrm{PIV}}(\mathrm{mm} / \mathrm{s})$ & $v^{\mathrm{NLR}+\mathrm{R}}(\mathrm{mm} / \mathrm{s})$ & $v^{\mathrm{R}}(\mathrm{mm} / \mathrm{s})$ \\
\hline 100 & 45 & 50 & 40 & 20 \\
200 & 89 & 100 & 80 & 40 \\
400 & 178 & 150 & 160 & 80 \\
\hline \hline
\end{tabular}

to a conservative $\sigma=10 \mathrm{~mm}$, yields that the Nonlinear radiation hypothesis has a plausibility $p(\mathrm{NLR})=0.9999918$ whereas the Reynolds stress has $p(\mathrm{R})=0.0000082$, in other words, an odds ratio of $10^{5}: 1$.

A Nonlinear radiation term arising in the Navier-Stokes equation has been derived, which was still now not believed to exist. It is a multiscale phenomenon responsible for generating a macroscopic stress field and an ensuing flow when an oscillating wave at the micro time scale, such as an ultrasonic wave, is present. This phenomenon is rooted in the constitutive nonlinearity of the fluid in the sense of deviation from Hooke's law, which is a direct consequence of the anharmonicity of the interatomic forces.

The range of validity of the NLR was verified experimentally for water ranging the following conditions: (i) a range of ultrasonic pressures up to $600 \mathrm{kPa}$, (ii) a range of ultrasonic frequencies between $3.75 \mathrm{MHz}$ and $4.25 \mathrm{MHz}$ given by the bandwidth of the used piezoelectric transducer, (iii) a range of acoustic fountains up to $20 \mathrm{~mm}$, above which they become unstable.

The author is grateful to Rafael Muñoz and END Lab team members for their constructive discussion, the Spanish Ministerio de Economía y Competitividad for Project DPI2010-17065, and Junta de Andalucía for Projects P11CTS-8089 and GGI3000IDIB.

${ }^{1}$ L. Rayleigh, Philos. Trans. R. Soc. 175, 239 (1883).

${ }^{2}$ C. Eckart, Phys. Rev. 73, 68 (1948).

${ }^{3}$ L. N. Liebermann, Phys. Rev. 75, 1415 (1949).

${ }^{4}$ J. J. Markham, Phys. Rev. 86, 497 (1952).

${ }^{5}$ W. L. Nyborg, J. Acoust. Soc. Am. 25, 68 (1953).

${ }^{6}$ P. Westervelt, J. Acoust. Soc. Am. 25, 60 (1953).

${ }^{7}$ S. J. Lighthill, J. Sound Vib. 61, 391 (1978).

${ }^{8}$ N. Riley, Theor. Comput. Fluid Dyn. 10, 349 (1998).

${ }^{9}$ N. Riley, Annu. Rev. Fluid Mech. 33, 43 (2001).

${ }^{10}$ S. Tjøtta, J. Sound Vib. 6, 255 (1967).

${ }^{11}$ S. Tjøtta, Ultrasonics 38, 278 (2000).

${ }^{12}$ T. Hasegawa, T. Kido, T. Iizuka, and C. Matsuoka, Acoust. Sci. Technol. 21, 145 (2000).

${ }^{13}$ F. A. D. H. C. Starritt and V. F. Humphrey, Ultrasound Med. Biol. 15, 363 (1989).

${ }^{14}$ A. Nowicki, W. Secomski, and J. Wojcik, Ultrasound Med. Biol. 23, 783 (1997).

${ }^{15}$ J. A. Cosgrove, J. M. Buick, S. D. Pye, and C. A. Greated, Ultrasonics 39, 461 (2001).

${ }^{16}$ T. Nowak, C. Cairos, F. Holsteyns, A. Lippert, and R. Mettin, in DAGA 2012 - Darmstadt, edited by H. Hanselka (H. Hanselka, 2012), pp. $457-458$.

${ }^{17}$ J. Koiller, K. M. Ehlers, and F. Chalub, Arbor, Ciencia, Pensamiento y Cultura Noviembre-Diciembre, 1089 (2010).

${ }^{18}$ S. J. Lighthill, J. Fluid Mech. 106, 149 (1981).

${ }^{19}$ T. M. Squires and S. R. Quake, Rev. Mod. Phys. 77, 977 (2005).

${ }^{20}$ J. Vanneste and O. Bühler, "Streaming by leaky surface acoustic waves," Proc. R. Soc. A (published online).

${ }^{21}$ P. B. Muller, "Acoustofluidics in microsystems: investigation of acoustic streaming," Master dissertation (Technical University of Denmark, 2012).

${ }^{22}$ H. Bruus, Lab Chip 12, 20 (2012).

${ }^{23}$ J. R. Doherty, G. E. Trahey, K. R. Nightingale, and M. L. Palmeri, IEEE Trans. Ultrason. Ferroelectr. Freq. Control 60, 685 (2013).

${ }^{24}$ J. E. Jones, Proc. R. Soc. London, Ser. A 106, 463 (1924).

${ }^{25}$ C. Pantea, C. F. Osterhoudt, and D. N. Sinha, Ultrasonics 53, 1012 (2013).

${ }^{26}$ B. T. Sturevant, C. Pantea, and D. N. Sinha, in Proceedings of IEEE International Ultrasonics Symposium (IEEE, 2012), pp. 284-287.

${ }^{27}$ See supplementary material at http://dx.doi.org/10.1063/1.4894827 for deriving the Nonlinear Radiation term in Navier-Stokes equation from the multiscale nonlinearity.

${ }^{28}$ L. D. Landau and E. M. Lifshitz, Theory of Elasticity, Course of Theoretical Physics Vol. 7 (USSR Academy of Sciences, 1959).

${ }^{29}$ J. W. S. Rayleigh, Theory of Sound (Macmillan, 1896).

${ }^{30}$ J. K. Kevorkian and J. D. Cole, Multiple Scale and Singular Perturbation Methods, Applied Mathematical Sciences (Springer, 1996). 